\title{
Interface Exchange as an Indicator for Eddy Heat Transport
}

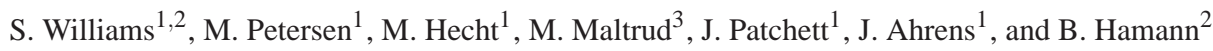 \\ ${ }^{1}$ Computer, Computational and Statistical Sciences Division, Los Alamos National Laboratory \\ ${ }^{2}$ Institute for Data Analysis and Visualization (IDAV), Department of Computer Science, University of California, Davis \\ ${ }^{3}$ Theoretical Division, Los Alamos National Laboratory
}

\begin{abstract}
The ocean contains many large-scale, long-lived vortices, called mesoscale eddies, that are believed to have a role in the transport and redistribution of salt, heat, and nutrients throughout the ocean. Determining this role, however, has proven to be a challenge, since the mechanics of eddies are only partly understood; a standard definition for these ocean eddies does not exist and, therefore, scientifically meaningful, robust methods for eddy extraction, characterization, tracking and visualization remain a challenge. To shed light on the nature and potential roles of eddies, we extend our previous work on eddy identification and tracking to construct a new metric to characterize the transfer of water into and out of eddies across their boundary, and produce several visualizations of this new metric to provide clues about the role eddies play in the global ocean.
\end{abstract}

Categories and Subject Descriptors (according to ACM CCS): Computer Graphics [I.3.8]: ApplicationsOceanography Simulation and Modeling [I.6.6]: Simulation Output Analysis—Ocean General Circulation Models

\section{Introduction}

Mesoscale eddies are vortices with diameters on the order of a hundred kilometers and typical lifetimes on the order of months. They are most often associated with the western boundary currents, and with the Antarctic Circumpolar Current, the unobstructed flow of fast-moving water in the Southern Ocean around Antarctica. These eddies are of interest to oceanographers because they can isolate heat, salt, and biological material and are capable of transporting materials in ways not accounted for by the large-scale circulation.

However, this paragraph asks more questions than it answers. What does it mean for eddies to isolate water? Is it like filling a huge balloon and towing it by ship to a destination? In a study analyzing the "gold standard" of eddiesspecial eddies called the Agulhas Rings, that are long-lived, isolated eddies generated by the Agulhas Current off the southern coast of Africa that then travel across the South Atlantic, sometimes reaching the coast of South America-it was found that the Agulhas Rings leak water at a rapid rate relative to their lifetime [dvD04]. This study raises this question of whether leakage rates are comparably high for other, less spectacular eddies.

Other issues have been left unaddressed as well. How much heat and salt transport are eddies responsible for, and where? Are there places where eddies are more important? This work provides a useful tool for analyzing these questions, and while this study does not provide complete answers to these questions, we will show and discuss these questions in several extensively-studied regions of the global ocean.

Our previous work [WPB* 11 ] was primarily concerned with extracting and tracking eddies from data generated by a high-resolution global ocean simulation. For this work, we put those abilities to use in order to fill in some of the gaps. To that end, we developed a metric to quantify leakage of a tracer variable (we focus on heat) into and out of eddies. Using this metric, we show the leakage profiles for some prominent individual eddies, and we aggregate this metric over time to show the patterns of heat transport in some eddyrich regions of the ocean. The corresponding visual analysis enabled by this method provides the first step toward a more rigorous and comprehensive analysis of eddy behavior.

\section{Related Work}

Extracting and visualizing turbulence in vector fields has long been of interest to the fluid dynamics and flow visualization communities [LHZP07]. Methods generally focus on either extracting specific structures (e.g., vortices) and 
drawing a bounding volume [SW97], or extracting the overall topology and visualizing it through glyphs or other proxies [TG09]. For extracting vortex-like structures in particular, standard methods include finding regions of high vorticity [VV92], streamline geometry [PKPH09], acceleration magnitude [KRHH11], or, when the data are available, by searching for regions of low pressure at the center of a vortex [BS94].

For vortices with core lines that are straight or nearly straight lines, as is the case in our application where vortex core lines are straight in depth direction, one can also look for circular behavior in the velocity field directly. Jiang et al. analyze the problem topologically [JMT02], by searching for kernels in which each vector in the kernel points in a unique direction range. Similarly, Sood et al. [SJBT05] identify vortex centers in the ocean by passing a five-by-five kernel of the angles between an east-pointing vector and the tangents of a circle centered in the kernel to find circular flows, then fitting an ellipse over the entire vortex.

Several techniques have also been developed to further refine analysis of the vorticity field. We use the $R^{2}$-criterion [WPB*11], a refinement of the $Q$ criterion [HWM88] (known as the Okubo-Weiss criterion [Oku70] in the oceanography community), which looks for vortices that resemble an idealized Gaussian vortex. Also of note is the $\lambda_{2}$-criterion [JH95], that uses the second eigenvalue of the strain tensor times the sum of the squares of strain and vorticity to determine whether vorticity or strain is dominant in a region. We chose the $R^{2}$-criterion over $\lambda_{2}$ because our work on $R^{2}$ showed that the Gaussian vortex model is a good fit for mesoscale eddies, so the $R^{2}$-criterion obtains a more refined selection of vortices.

In oceanography, numerous studies of satellite data have quantified the size and distribution of oceanic eddies, mostly based on consideration of the Okubo-Weiss parameter. Published works have considered regions including the Mediterranean Sea [IFGLFGO06], the Tasman Sea [WAB06], the Gulf of Alaska [HT08] and the nearly-global domain of all but the ice-covered seas [CSSdS07, CSS11].

An eddy census based on satellite data is limited to surface features, while data from vertical ship-deployed profiles and fixed moorings are sparse and can only capture an incomplete picture. Ocean models have been used as a means to study features that are not directly observable at this time, and therefore provide a means for filling in our understanding of the oceans. Much of the knowledge of eddy transports of heat, salt and nutrients has been derived from the analysis of ocean models [JM02, YNQ*10]. It is important to note that the discussion of eddy transport in the literature generally refers to what we call variability transport, which is to say, all transport not accounted for in the mean flow, which will include any temporal variability in the mean flow, such as the slow meandering of large-scale currents.

\section{Background}

There are two important piece of background that will be needed for the development of our interface exchange metric. On the oceanographic side, we will briefly introduce tracer transport in fluid dynamics, so that it is clear what we mean by transport and so that the mathematical foundations are in place for the development of our new metric. On the visualization side, we will summarize previous computational work, covering how we extract and track eddies, and some relevant details about the simulation from which our data originate.

\subsection{Aggregate Transport}

In order to develop an understanding of eddy transport, we must first cover some background material from fluid dynamics; see [WF11] for more complete coverage of the topic. Tracer transport is defined as $\mathbf{u} T$, where $\mathbf{u}$ is velocity and $T$ is a scalar quantity such as temperature. Scalars that can be advected by fluid flow are called "tracers," due to the fact that they can be used to track fluid flow by tracing it out. (This is not entirely true for "active tracers," that also influence flow by altering the dynamic characteristics of the fluid, such as the ability of temperature to affect pressure, but the name is a reasonable approximation.) In the ocean, the most common tracers are temperature and salinity; we will focus on temperature, but any discussions, equations, and results in this paper apply to any tracer.

The most common decomposition of transport is to separate it into mean transport - transport caused by time mean circulation, such as the clockwise circulation of water over the midlatitude North Atlantic Ocean-and what is commonly called eddy transport. Eddy transport, in this decomposition, is any transport not accounted for by time mean circulation, which includes eddies, but also includes all other time-varying flow. To make a distinction between transport actually caused by eddies and that caused by all sources of variability in the flow, we will refer to the latter as variability transport.

Mean velocity, defined as $\overline{\mathbf{u}}$, is computed by taking the component-wise time mean over some temporal window of velocity. (We use one-month averages, though it is also standard to consider yearly averages.) Variability velocity, defined as $\mathbf{u}^{\prime}$, can then be computed by subtracting the mean velocity from the instantaneous velocity: $\mathbf{u}^{\prime}=\mathbf{u}-\overline{\mathbf{u}}$. To state it as a decomposition, velocity is its time mean plus variability, or, $\mathbf{u}=\overline{\mathbf{u}}+\mathbf{u}^{\prime}$. Decomposing transport into mean and variability components then requires multiplying this decomposition of velocity with the same decomposition of the tracer:

$$
\begin{aligned}
\mathbf{u} T & =\left(\overline{\mathbf{u}}+\mathbf{u}^{\prime}\right)\left(\bar{T}+T^{\prime}\right) \\
& =\overline{\mathbf{u}} \bar{T}+\mathbf{u}^{\prime} \bar{T}+\overline{\mathbf{u}} T^{\prime}+\mathbf{u}^{\prime} T^{\prime}
\end{aligned}
$$



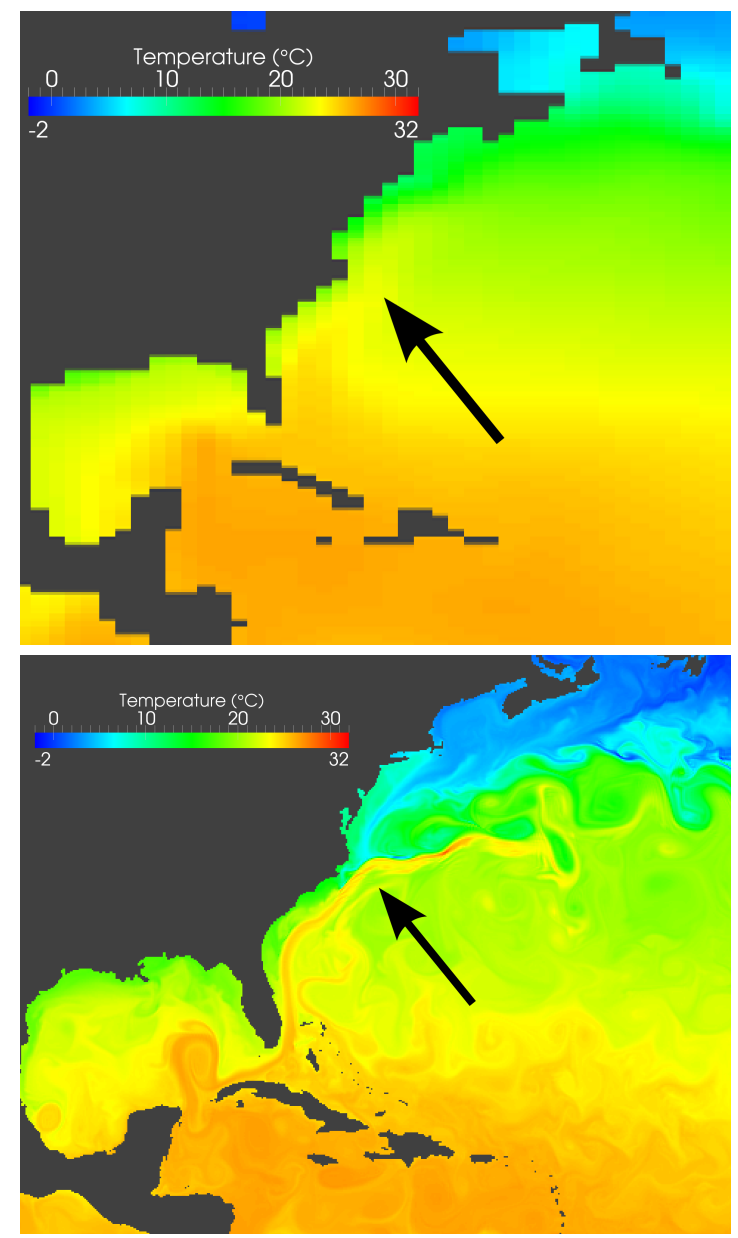

Figure 1: Sea surface temperature in the vicinity of the Gulf Stream, computed by two different models. The top plot is from a simulation with a spatial resolution of $1^{\circ}$, while the bottom is made from POP, at a spatial resolution of $\frac{1}{10}^{\circ}$. In both images, the Gulf Stream itself appears as a yellowish line coming north from the Gulf of Mexico. In the highresolution simulation, the Gulf Stream departs the coast of the United States near the state of North Carolina, while in the low-resolution simulation, the Gulf Stream continues hugging the coast of the United States. This incorrect path of the Gulf Stream is a well-known problem with low-resolution simulations. One important difference between a $1^{\circ}$ model and $a \frac{1}{10}^{\circ}$ model is that the former does not have sufficient resolution for eddies to appear, hence, the emergence of eddies is a possible cause for higher-resolution simulations producing the Gulf Stream with the correct shape.
Oceanographers are often more interested in aggregate behavior, since the behavior at any particular moment in time may not be indicative of the general functioning of the ocean. Aggregate transport is computed by taking its time mean:

$$
\begin{aligned}
\overline{\mathbf{u} T} & =\overline{\overline{\mathbf{u}} \bar{T}+\mathbf{u}^{\prime} \bar{T}+\overline{\mathbf{u}} T^{\prime}+\mathbf{u}^{\prime} T^{\prime}} \\
& =\overline{\overline{\mathbf{u}} \bar{T}}+\overline{\mathbf{u}^{\prime} \bar{T}}+\overline{\overline{\mathbf{u}} T^{\prime}}+\overline{\mathbf{u}^{\prime} T^{\prime}}
\end{aligned}
$$

This equation can be simplified via two observations. First, $\overline{\mathbf{u}^{\prime}} \bar{T}=\overline{\mathbf{u}^{\prime}} \bar{T}$, because $\bar{T}$ is constant over the averaging window, since it has already been averaged, and constants can be pulled out of averages. Also, $\overline{\overline{\mathbf{u}}} \bar{T}=\overline{\mathbf{u}} \bar{T}$ for the same reason. Second, $\overline{\mathbf{u}^{\prime}}=0$, because, when using the time average of $\mathbf{u}=\overline{\mathbf{u}}+\mathbf{u}^{\prime}$, we obtain $\overline{\mathbf{u}}=\overline{\mathbf{u}}+\overline{\mathbf{u}^{\prime}}$, meaning that $\overline{\mathbf{u}^{\prime}}$ must be zero. (The same logic applies to $\overline{\overline{\mathbf{u}} T^{\prime}}$.) This simplifies the equation to the canonical expression of time-average tracer transport:

$$
\overline{\mathbf{u} T}=\overline{\mathbf{u}} \bar{T}+\overline{\mathbf{u}^{\prime} T^{\prime}}
$$

Here, $\overline{\mathbf{u}} \bar{T}$ is the mean transport, while $\overline{\mathbf{u}^{\prime} T^{\prime}}$ is the variability transport.

There are two ways in which we intend to extend this analysis. The first is that, as noted above, this definition of variability transport includes all time-varying flow, while we would like to study eddies in particular. Second, we would like to know specifically where eddies are important. To explain this point further, in Figure 1, we show two images of the Gulf Stream. The top image is from a $1^{\circ}$ simulation, while the bottom is from a $\frac{1}{10}^{\circ}$ simulation. The higherresolution data agree fairly well with satellite observation of the North Atlantic: the Gulf Stream (a yellowish line in these images) travels north from the Gulf of Mexico, then heads offshore near the state of North Carolina. In the lowresolution simulation, however, the Gulf Stream continues to hug the coast even after North Carolina. The relevance of this comparison is that $\frac{1}{10}^{\circ}$ is high enough resolution for mesoscale eddies to appear, while $1^{\circ}$ is not. In other words, it is possible that the heat transport caused by eddies is a factor in producing the correct Gulf Stream shape, hence we would like to know if eddy transport of heat is especially important in that region.

\subsection{Eddy Detection and Tracking}

Studying eddies in particular requires a reasonable definition of eddies. To this end, we employed a simplified version of the $R^{2}$-criterion [WPB*11]. One of the questions that motivated the $R^{2}$ work was the arbitrariness of taking thresholds of the $Q$-criterion. When oceanographers use the $Q$-criterion to identify eddies, a common standard is to normalize $Q$ by its standard deviation, i.e., $Q_{\text {norm }}=\frac{Q}{\sigma_{Q}}$, and to 
use a threshold of $Q_{n o r m} \geq 0.2$ to identify eddies. Presumably, this threshold is chosen because it extracts features that look like what an oceanographer expects eddies to look like. In applying the $R^{2}$-criterion to the global ocean and choosing the maximum $Q$ value that met a confidence level of 0.9 , we found that, in general, $Q_{\text {norm }} \geq 0.2$ is very close to the maximum possible threshold that meets an $R^{2}$ confidence of 0.9 .

Keeping this in mind, the simplification we employed uses the same $Q$ threshold, $Q_{n o r m} \geq 0.2$, but we only keep features when they satisfy $R^{2} \geq 0.9$. That is, for each feature, we compare the quality of a linear fit for area versus $Q$ for several level sets of $Q$, and only call that feature an eddy if the quality of the fit (the $R^{2}$ value) is at least 0.9 .

In order to track eddies over time, we begin by attempting to predict where each eddy will be in the next several time steps, and when an eddy appears where we predict a previous one should be, we say they represent the same eddy at different points in time. The prediction is based on considering an eddy as a spinning hockey puck sliding across an ice rink. This puck has two major velocities superimposed on each other: an angular velocity representing the spinning, and a linear velocity representing the translation. If the puck were merely spinning in place (or if we went to a frame of reference in which the puck is spinning in place), then its average linear velocity would be zero, because angular components on opposite sides of the puck cancel each other out. If we consider a reference frame where the puck is also sliding, only the sliding velocity will contribute to the puck's average velocity, since the contribution to average velocity from its spinning will still be zero. Thus, we approximate an eddy's translational velocity as its average velocity. Each eddy from an immediately previous time step is translated to its expected new position, and if an eddy resides near the predicted position, the two eddies are connected in a tracking graph. Because eddies move relatively small distances between time steps-the center of an eddy generally only moves one or two voxels per time step-the tracking is quite stable.

\subsection{Data Source}

Our simulation data come from global runs of the Parallel Ocean Program (POP) [SDM92]. The data have a spatial resolution of about $\frac{1}{10}^{\circ}$ and a temporal resolution of one day. A principal "input" to POP is surface heat flux; for fullycoupled simulation runs, POP is run along with an atmospheric simulation, and the two trade information across the sea surface via heat fluxes. The run that generated the data we employed was not fully-coupled, so POP was run in isolation. These runs instead use estimated surface flux values (meaning that the simulation lacks atmospheric feedback), including seasonal flux variation. In order to avoid averaging across seasons, we use data from one spring season, which we take to be the months of March, April, and May.

\section{Interface Exchange}

With all this in place, we now move on to characterizing eddy heat transport. There are essentially three hypotheses to describe the general behavior of eddies: (i) eddies completely trap water within their boundary, which is only released when the eddy is destroyed; (ii) eddies are a temporary distortion of the column of water they occupy, but do not trap water at all; and (iii) eddies do trap water, but they also actively exchange it across their boundaries. We call these, respectively, the sealed container hypothesis, the wave phenomenon hypothesis, and the leaky container hypothesis.

We initially tried to evaluate these hypotheses through direct study of temperature data from POP. Using our eddy tracking system, we found several of the longest-lived eddies and extracted distributions of the temperature of water inside and directly outside the eddy over each eddy's entire lifetime, and at several depths. The boundary of the eddy is defined by a contour of the $Q$-criterion (with $Q_{\text {norm }}=0.2$ ), hence the inside of the eddy is defined as the inside of that contour, or, points with $Q_{n o r m} \geq 0.2$ associated with that particular contour. To define the exterior of the eddy, we take a bounding box concentric with the eddy and with edge length twice the diameter of the eddy, and consider all points that cannot be inside an eddy, i.e., we consider only points with $Q_{\text {norm }}<0.2$.

Among the eddies extracted by this method, we chose three representative eddies, shown in figure 2. Eddies are known to be able to raise and lower a water column, and since temperature is related to depth in the ocean, the offset between inside and outside temperature (i.e., the eddies are mostly either colder or hotter than outside water, and that offset is consistent over time) is expected. This contrast in temperature between waters inside and outside of the eddy is part of what establishes the pressure gradient that supports the eddy (this is called geostrophic balance [Val06]). Consequently, dynamical adjustment makes it problematic to determine whether the temperature contrast is maintained through minimal leakage, or whether more significant leakage through the lateral boundary of the eddy is being balanced by vertical motion sufficient to maintain the temperature contrast across the boundary.

In order to introduce a better conceptual model to understand the three eddy behavior hypotheses, consider a thought experiment in which a passive tracer (i.e., a scalar that is advected by water flow, but its concentration does not affect the other properties of the water in any way) is inserted into an eddy with a concentration of one, while the external waters have a concentration of zero. If the eddy is best approximated as a sealed container, the dye would simply move along with the eddy, and not contaminate the external waters; if eddies are only wave phenomena, the eddy would propagate away but the dye would remain behind, being advected and sheared by the mean flow but with no strong relationship to the eddy. In between is the leaky container hy- 
S. Williams, M. Petersen, M. Hecht, et al. / Interface Exchange as an Indicator for Eddy Heat Transport
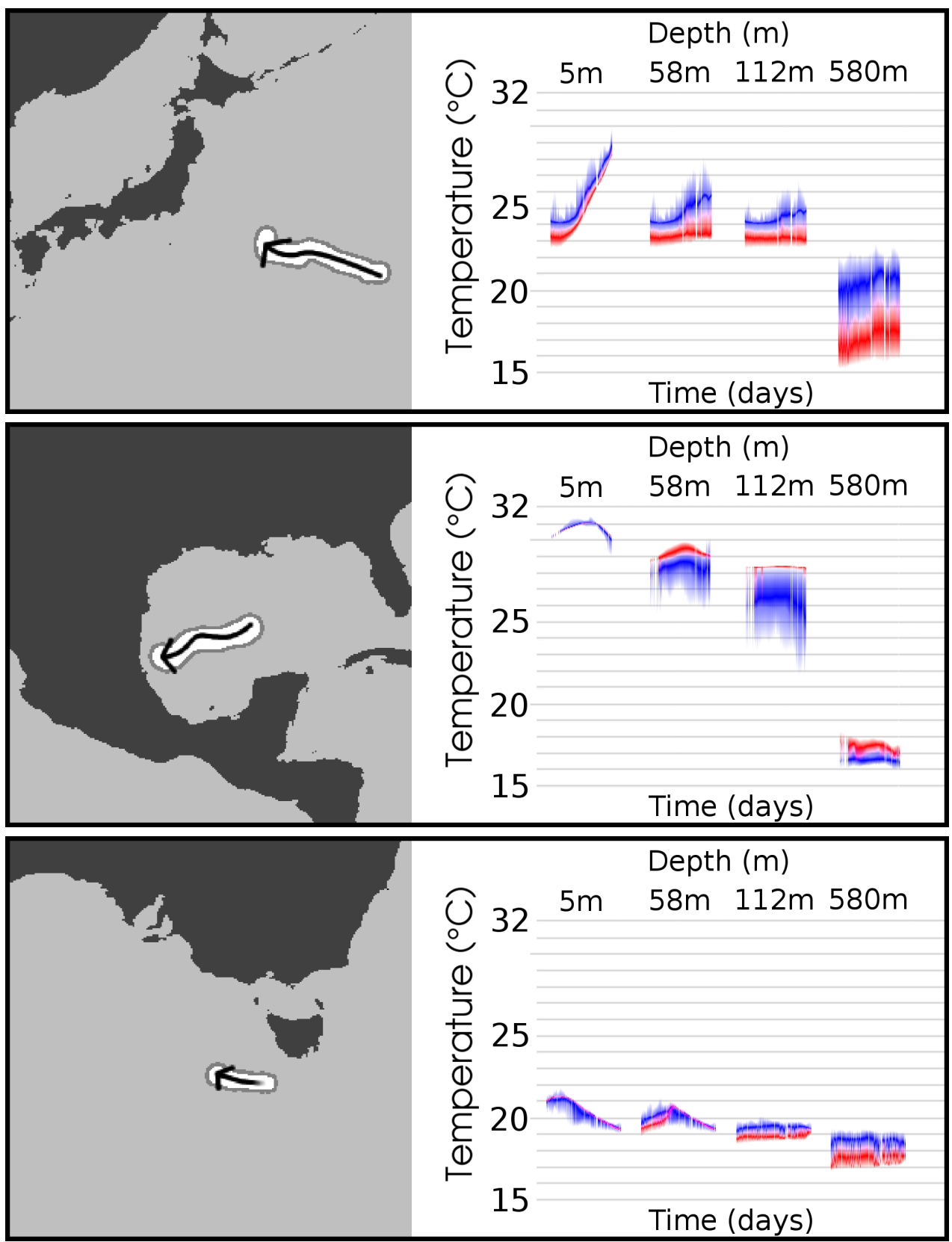

Figure 2: Inside and outside temperatures of eddies. Using both eddy identification and tracking, we computed the distribution of temperature both inside (red) and outside (blue) three eddies from different locations around the world. Also, quartile distance is mapped to alpha, with the median at full opacity, the lower and upper quartiles at 50\% opacity, and the minimum and maximum values at no opacity. Thus, where the two distributions overlap, they appear magenta. The temperature distributions for each eddy are shown at depths of $5 \mathrm{~m}, 58 \mathrm{~m}, 112 \mathrm{~m}$, and $580 \mathrm{~m}$, in order to make sure that the results are not skewed by differences between surface behavior, mixed layer behavior, and the deep ocean. The relationships that are seen between temperatures inside and outside of the eddy must be maintained in order to preserve the dynamics that support the eddy (as explained further in the text), but it is unclear whether this is primarily due to containment of waters inside the eddy, or whether substantial leakage is compensated by vertical motion of the water column. In the particular case of the Australian eddy at a depth of 58m, these effects are confounded by fluctuations in the mixed layer depth (the upper layer of the ocean, characterized by large amounts of turbulent mixing) that are known to occur in this region. Thus, we need a better metric to help to distinguish between these scenarios. 
pothesis, where the dye is partially taken with the eddy, but diffuses out of the eddy boundary along the way.

Indeed, a passive tracer study to that effect has been carried out [dvD04]. In this study, simulated eddies in the South Atlantic, named Agulhas Rings after the current that spawns them, were seeded with a passive tracer. Over time, water leaked out of the eddies, indicated by the tracer appearing outside the eddy. However, water initially inside the eddy could remain in the eddy for several weeks, indicated by the tracer remaining inside the eddy in nontrivial concentrations. Thus, the rest of our efforts are devoted to the explicit diagnosis of the fluxes in and out of eddies that would be expected by the leaky container hypothesis.

To determine how much water from eddies are leaking and where, we must determine the velocity of water perpendicular to the interface between the eddy and the background water. This follows from the fact that, at the interface, water that is trapped by the eddy will be going along the interface; the only direction by which water can leave the eddy is the direction normal to the interface. To accomplish this, we developed a decomposition of velocity that allows us to remove every term except for that normal to the interface.

The first step is to create a reference frame in which the eddy is stationary. The simulation uses a reference frame in which the Earth is stationary, so making the eddy stationary requires subtracting any velocity that moves with respect to the Earth. There are two such sources of velocity: the largescale mean flow, and the translation of the eddy itself. The former can be computed by taking a time average of velocity, as in Section 3.1. The latter can be computed by taking the average velocity of the eddy (averaged in space for a single time step), as argued in Section 3.2, where the argument was made for establishing the translational velocity of the eddy for the purpose of tracking.

With the eddy placed in a reference frame in which it is stationary, the last component of velocity to remove is circulation inside the eddy. Since we are concerned only with the boundary of the eddy, what we really need to remove are components of velocity pointing along the boundary, which we define as a contour of the $Q$-criterion. The direction perpendicular to the boundary, then, is defined by the gradient of $Q$. To obtain only the velocity pointing along the gradient of $Q$, we simply take a dot product.

These four components are shown schematically in Figure 3. By removing all of them except the boundary normal component, the interface exchange is defined as:

$$
\begin{aligned}
\mathbf{u} T_{i}= & \left(\left(\left(\mathbf{u}-\overline{\mathbf{u}}^{t}\right)\left(T-\bar{T}^{t}\right)-\right.\right. \\
& \frac{\left.\left.\left(\mathbf{u}-\overline{\mathbf{u}}^{t}\right)\left(T-\bar{T}^{t}\right)^{e}\right) \cdot \frac{\nabla Q}{\|\nabla Q\|}\right) \frac{\nabla Q}{\|\nabla Q\|}}{}
\end{aligned}
$$
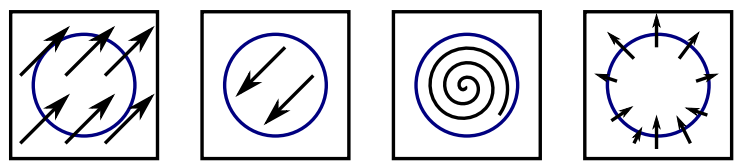

Figure 3: Decomposition of the velocity field inside an eddy: the eddy is imposed on a background flow, the core of the eddy is translating, water inside the eddy is circulating, and exchange is occurring along its boundary.

$$
=\left(\left(\mathbf{u}^{\prime} T^{\prime}-\overline{\mathbf{u}^{\prime} T^{\prime}}{ }^{e}\right) \cdot \frac{\nabla Q}{\|\nabla Q\|}\right) \frac{\nabla Q}{\|\nabla Q\|}
$$

Here, a bar with a superscripted $t$ indicates a pointwise average over time, while a bar with a superscripted $e$ indicates a spatial average over the entire eddy within a single time step. The final multiplication by $\nabla Q$ indicates the direction in which the transport is taking place, since the dot product with $\nabla Q$ will produce a scalar. This allows us to aggregate the interface transport over time, and have transports in opposing directions cancel each other out.

\section{Results}

The instantaneous interface exchange is shown in Figure 4 for three prominent eddies. From top to bottom, they are an Agulhas Ring, a Loop Current Ring, and a Gulf Stream Ring. The Agulhas Ring and the Loop Current Ring both show prominent quadrants directed oppositely to each other: both eddies take in heat from the northwest and southeast, and expel heat to the southwest and northeast. The Gulf Stream Ring, on the other hand, appears to be undergoing deformation from the south (the camera in this image is facing west), and the eddy is moving a significant amount of heat across its boundary at the deformation. This suggests that deformation may be a powerful mechanism by which eddies exchange heat with their surroundings.

In addition to the instantaneous behavior of the interface exchange metric, we would like to know about the aggregate effect of eddies. For this, we time-integrated the interface transport field over the three springtime months. In general, this field turned out to be quite chaotic, but there are some locations calm enough to visualize with glyphs. In Figure 5, we show the vicinity of the same Agulhas Ring and the Loop Current Ring, aggregated over three months. These images are also overlaid on a backdrop of the instantaneous $Q$ field from April 15 (of the second year of the simulation), to provide a reference field. (Since the overlay is an aggregate field on an instantaneous field, there is little alignment between the two.) We see that the behavior of the Agulhas Ring is extremely consistent: the Agulhas Rings, over fairly long time scales, take in water from the southeast and northwest, and expel water to the west and east. The Loop Current Ring, on the other hand, moves somewhat chaotically, so even though 

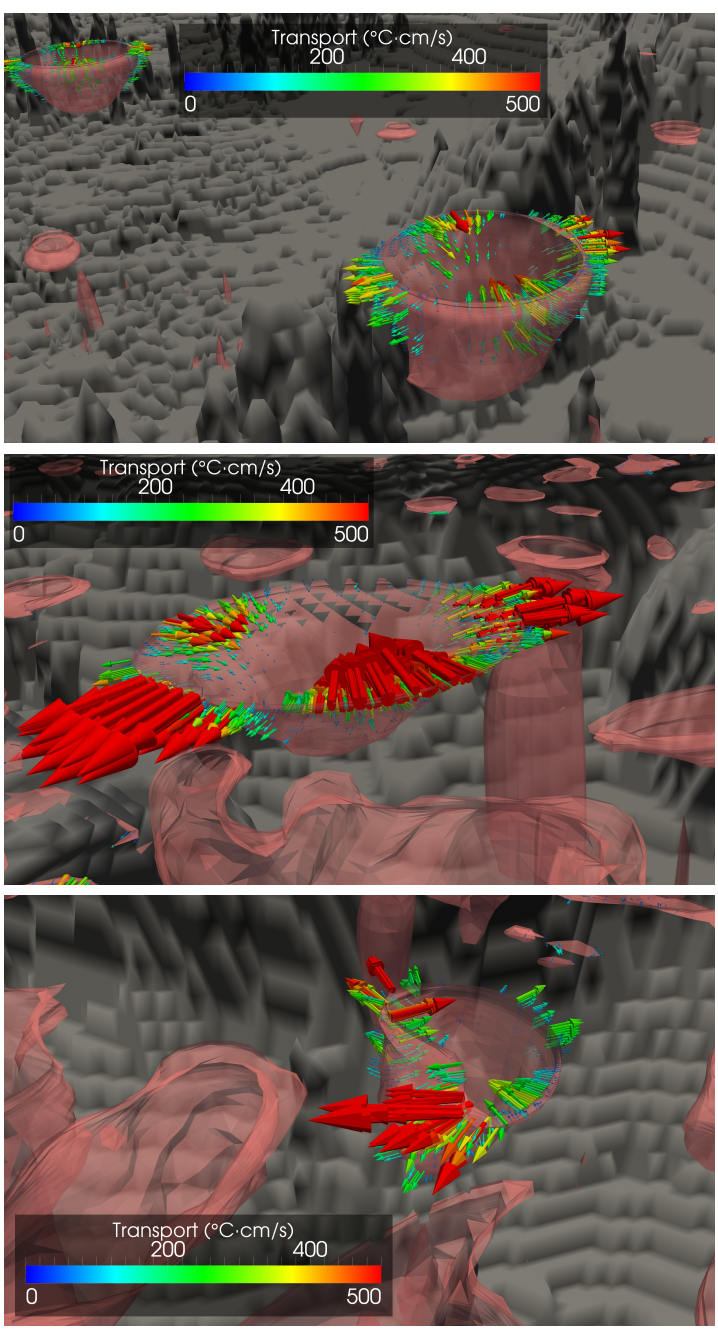

Figure 4: Close-up visualizations of interface exchange behavior. We apply the interface exchange metric to a single time step, focusing on three eddies engaging in particular strong interface exchange at this point in time. The red surfaces are contours of the $Q$-criterion, so they are what we consider the eddy boundaries, while arrows indicate the magnitude (in both color and arrow size) and direction of heat transport. The three eddies pictured are, from top to bottom: an Agulhas Ring, a long-lived eddy moving west across the South Atlantic from the southern tip of Africa; a Loop Current Ring, a long-lived eddy generated in the Gulf of Mexico; and a Gulf Stream Ring, a short-lived eddy associated with the Gulf Stream in the North Atlantic. The image of the Agulhas Ring has a camera oriented in the northern direction; the image of the Loop Current Ring is looking northwest toward the US state of Texas; and the image of the Gulf Stream Ring is oriented in the western direction. The Agulhas Ring and Loop Current Ring appear to be contributing to a long-term, stable heat transport, see also Figure 5, while the Gulf Stream Ring appears to be contributing to heat transport primarily where it undergoes deformation, when large exchanges of heat may occur.

(c) 2012 The Author(s)

(c) 2012 The Eurographics Association and Blackwell Publishing Ltd.
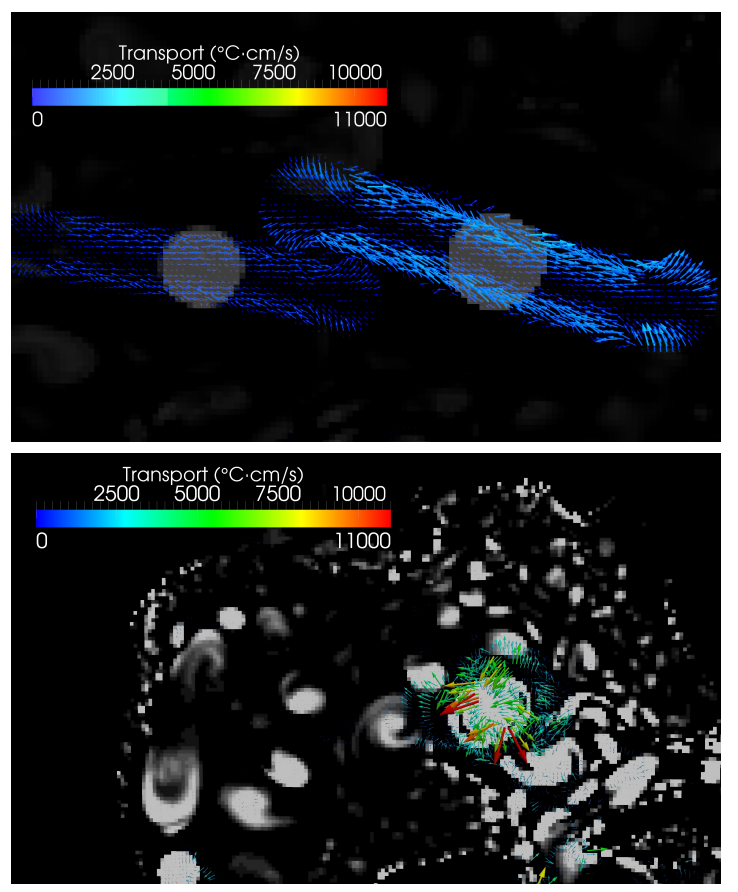

Figure 5: Stable and chaotic behavior of Agulhas Rings and a Loop Current Ring. After applying time aggregation, the interface transport field is too chaotic in most locations for effective visualization using glyphs. However, the Agulhas Rings (top) and Loop Current Ring (bottom) are sufficiently powerful and isolated so that their effects dominate the interface transport in their vicinity of the ocean. Both images are overlaid on a snapshot of the $Q$-criterion from the middle of the time range (specifically, April 15), but these overlays are only meant to serve as a visual reference. The Agulhas Ring shows a remarkably stable pattern, pulling in heat from the southeast and pushing it out to the west, and pulling in heat from the northwest and pushing it out to the east. The Loop Current Ring is far more chaotic, though in general it seems to be transporting heat to the southwest, against the usual clockwise flow of water along the edge of the Gulf of Mexico.

it has a strong tendency to flux heat to the southwest, it does not do so in an orderly manner.

For our final visualization of interface exchange, we used the three-month sum of interface exchange, and downsampled it by a factor of ten in order to bring out the dominant trends. We visualized the exchange metric in Figure 6 using streamtubes with radius proportional to transport magnitude, and with directed cones on the streamtubes indicating the direction in which transport is taking place. In the vicinity of the Gulf Stream, the interface exchange metric indicates that eddies are primarily moving heat westward, against the eastward flow of the Gulf Stream. As indicated above, in the 
Gulf of Mexico, the interface exchange metric shows a predominantly southwest transport of heat. In the vicinity of the Kuroshio Current off the coast of Japan, the eddies seem to lack a primary direction of heat transport, though they do appear to follow along contours of the background temperature field.

The time-averaged eddy interface exchange (figure 6) is useful to see the locations where eddies consistently transport heat over long periods of time. For example, the Loop Current in the Gulf of Mexico is a warm ocean current that flows northward between the Yucatan Peninsula and Cuba, and then loops east and then south, and eventually leaves the Gulf through the Florida Straits. The Loop Current is clearly seen in figure $1 \mathrm{~b}$ as the orange ellipse west of Florida. The Loop Current pinches off to shed an eddy about twice each year [Oey04]. These eddies, called Loop Rings, always occur at the same location. This Loop Ring, that is pictured in figure $4 \mathrm{~b}$, is also visible in the time-averaged images in figures $5 \mathrm{~b}$ and $6 \mathrm{a}$. The vectors indicate that the heat transport across the eddy boundary is westward, counter the the mean current which is eastward in the central Gulf. Eddies advecting chaotically in the Gulf of Mexico do not stay still long enough to accumulate fluxes in time-averaged images (figures 5b, 6a).

Agulhas Rings are the most prominent of the ocean's eddies; they are $100-400 \mathrm{~km}$ in diameter, and travel from their formation region south of Africa across the South Atlantic. On average six Agulhas Rings cross the Atlantic each year, and half of them reach the South American coast. They are responsible for a significant fraction of the exchange from the Indian to the Atlantic Ocean [RLB03]. Because Agulhas Rings are well-separated as they travel through the quiescent South Atlantic, they are ideal test cases for analysis and visualization of eddies. The two structures visible in the single-time image in figure 4 clearly show the fluxes across the boundaries at this instant. Averaging over a short period of time, one sees a "railroad track" pattern in figure 5, because the perpendicular components of the fluxes at the front and back eventually cancel each other out.

The Gulf Stream and Kuroshio currents are powerful boundary currents with an average velocity of $1.5 \mathrm{~m} / \mathrm{s}$ and a maximum velocity of $2.5 \mathrm{~m} / \mathrm{s}$. These energetic jets meander and pinch off eddies on both sides. Transport across the eddy boundary is easily seen in the single-time image of a Gulf Stream Ring (figure $4 \mathrm{c}$ ). With time averaging, the aggregate image shows that eddies transport heat down gradient from warm to cold waters. This is consistent with previous studies of eddy heat transport in the Gulf Stream [WWH07]. Areas of high eddy interface exchange occur in energetic regions of the jets, indicating that eddies either remain in those positions for long periods, or eddies are consistently created at those positions, so that boundary exchange accumulates in the same locations.

\section{Conclusion}

We have described a new way to characterize and analyze ocean eddies via a metric for exchange across their boundaries. We have described how our data analysis and visualization system can be used to study specific scientific questions concerning the nature and role of eddies in the global ocean. Based on our earlier research on eddy identification and tracking, we have demonstrated that eddies may be important in certain key regions of the ocean. A major contribution of our effort, as far as data analysis and visualization science is concerned, is the successful design and implementation of a system allowing ocean modelers to more efficiently and more soundly test hypotheses and gain physical understanding from massive, complex simulated data sets.

Considering the caveats involved with using active tracers (such as temperature) to study water containment in eddies, we believe this application would be particularly powerful if used to study passive tracer leakage. Additionally, this work only covers transport of eddies during their lifetime. Another possible avenue for future work would look at the characteristics of eddies when they are created and destroyed.

This research is preliminary to a more rigorous analysis of eddies. This metric, along with our recently developed eddy extraction and tracking techniques, provide a basis with which to perform such an analysis, as we work towards a more comprehensive understanding of the role of eddies in the global ocean.

\section{References}

[BS94] Banks D. C., Singer B. A.: Vortex tubes in turbulent flows: identification, representation, reconstruction. In VIS ' 94 : Proceedings of the conference on Visualization '94 (Los Alamitos, CA, USA, 1994), IEEE Computer Society Press, pp. 132139. 2

[CSS11] Chelton D. B., Schlax M. G., Samelson R. M.: Global observations of nonlinear mesoscale eddies. Progress in Oceanography 91 (Oct. 2011), 167-216. 2

[CSSdS07] Chelton D. B., Schlax M. G., Samelson R. M., DE SzOEKE R. A.: Global observations of large oceanic eddies. Geophysical Research Letters 34, L15606 (2007), 5. 2

[dvD04] De Steur L., VAn LeEuwen P. J., Drijfhout S. S.: Tracer Leakage from Modeled Agulhas Rings. Journal of Physical Oceanography 34 (2004), 1387. 1, 6

[HT08] Henson S. A., Thomas A. C.: A census of oceanic anticyclonic eddies in the gulf of alaska. Deep Sea Research Part I: Oceanographic Research Papers 55, 2 (2008), 163 - 176. 2

[HWM88] Hunt J. C. R., Wray A. A., Moin P.: Eddies, streams, and convergence zones in turbulent flows. In Studying Turbulence Using Numerical Simulation Databases, 2 (Dec. 1988), pp. 193-208. 2

[IFGLFGO06] ISERN-FONTANET J., GARCÍA-LAdONA E., FOnT J., GARCÍA-Olivares A.: Non-gaussian velocity probability density functions: An altimetric perspective of the mediterranean sea. J. Phys. Oceanogr.36, 11 (2006), 2153 - 2164. 2

[JH95] JiNHEE JEONG, HUSSAIN F.: On the identification of a vortex. Journal of Fluid Mechanics 285 (25 February 1995), 6994. 2 

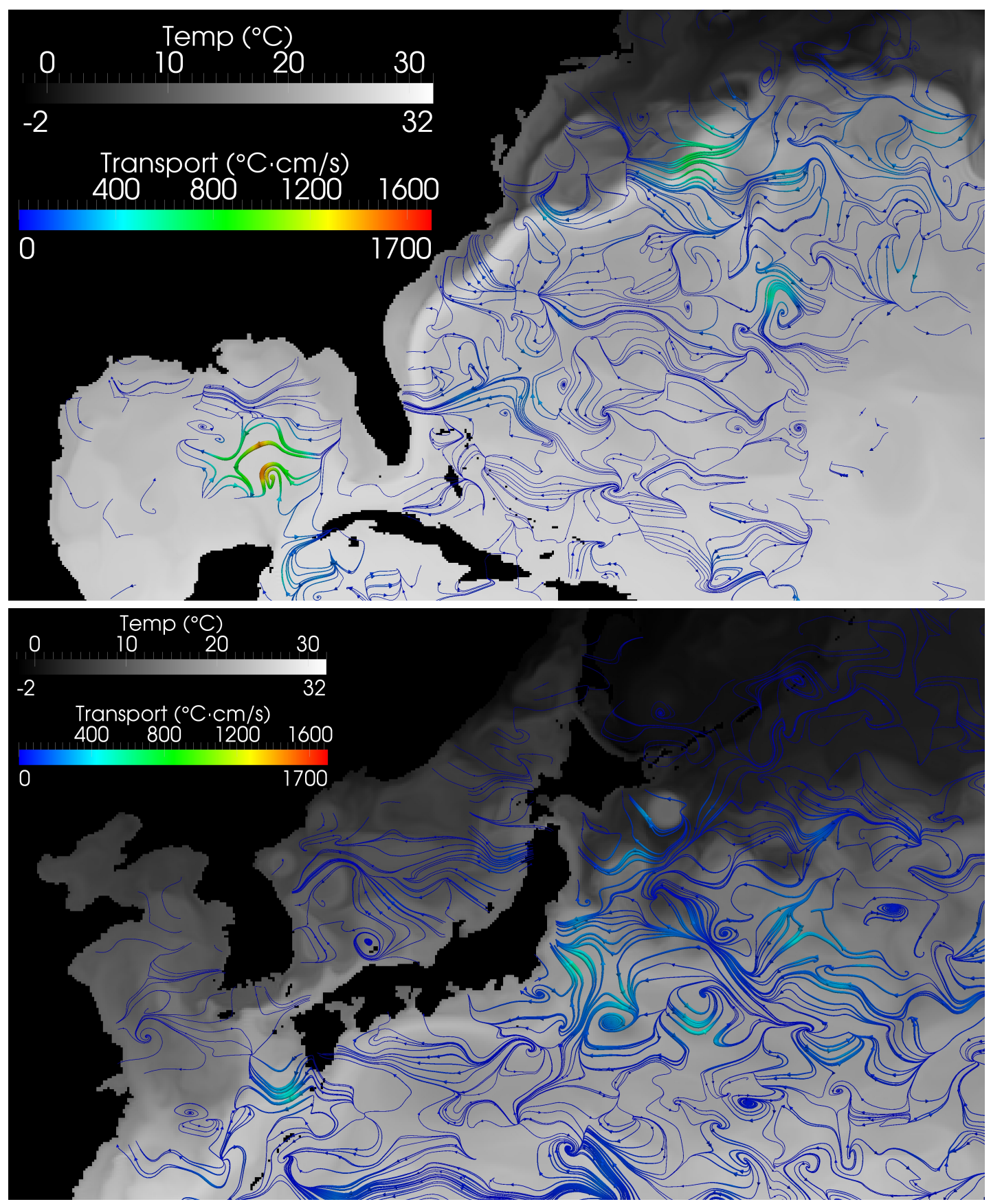

Figure 6: Interface exchange aggregation. After aggregating the interface exchange over three months, we downsample it by a factor of ten in order to bring out the dominant trends. These trends are then visualized with streamtubes, with radius proportional to the transport magnitude. Additionally, directed cones on the streamtubes indicate the direction that transport is occurring. The images are also overlaid on the temperature field averaged over the month of April, in order to provide a reference map. In the vicinity of the Gulf Stream, interface exchange is primarily moving water to the west, opposite to the eastward flow of the Gulf Stream. In the Gulf of Mexico itself, the eddies are moving water to the southwest, against the dominant flow of heat through the Gulf. The behavior near the Kuroshio Current, off the coast of Japan, is less straightforward. Eddies do, however, appear to be pushing heat along contours of heat, indicating they may be involved in shaping those contours. It should also be noted that these images only consider the ocean surface, where wind and solar forcing are in effect. 
[JM02] JAYNE S. R., MARotzke J.: The oceanic eddy heat transport*. Journal of Physical Oceanography 32, 12 (2002), 3328-3345. 2

[JMT02] Jiang M., Machiraju R., Thompson D.: A novel approach to vortex core region detection. In Proceedings of the symposium on Data Visualisation 2002 (Aire-la-Ville, Switzerland, Switzerland, 2002), VISSYM ’02, Eurographics Association, pp. 217-ff. 2

[KRHH11] Kasten J., Reininghaus J., Hotz I., Hege H.C.: Two-dimensional time-dependent vortex regions based on the acceleration magnitude. Visualization and Computer Graphics, IEEE Transactions on 17, 12 (dec. 2011), 2080 -2087. 2

[LHZP07] Laramee R., Hauser H., ZhaO L., Post F.: Topology-based flow visualization, the state of the art. In Topology-based Methods in Visualization, Hauser H., Hagen H., Theisel H., (Eds.), Mathematics and Visualization. Springer Berlin Heidelberg, 2007, pp. 1-19. 10.1007/978-3-540-708230_1. 1

[Oey04] OEY L.-Y: Vorticity flux through the Yucatan Channel and Loop Current variability in the Gulf of Mexico. Journal of Geophysical Research (Oceans) 109, C18 (Oct. 2004), 10004. 8

[Oku70] ОкUво A.: Horizontal dispersion of floatable particles in the vicinity of velocity singularities such as convergences. Deep Sea Research and Oceanographic Abstracts 17, 3 (1970), $445-454.2$

[PKPH09] Petz C., Kasten J., Prohaska S., Hege H.-C.: Hierarchical vortex regions in swirling flow. Computer Graphics Forum 28, 3 (2009), 863-870. 2

[RLB03] Richardson P., LutJeharms J., Boebel O.: Introduction to the "Inter-ocean exchange around southern Africa". Deep Sea Research Part II: Topical Studies in Oceanography 50 (Jan. 2003), 1-12. 8

[SDM92] Smith R. D., Dukowicz J. K., Malone R. C.: Parallel ocean general circulation modeling. Physica D: Nonlinear Phenomena60 (1992), 38-61. 4

[SJBT05] SOOD V., JOHN B., BALASUBRAMANIAN R., TANDON A.: Segmentation and tracking of mesoscale eddies in numeric ocean models. In Image Processing, 2005. ICIP 2005. IEEE International Conference on (September 2005), vol. 3, pp. III - 469-72. 2

[SW97] SILVER D., WANG X.: Tracking and visualizing turbulent $3 \mathrm{~d}$ features. Visualization and Computer Graphics, IEEE Transactions on 3, 2 (apr. 1997), 129-141. 2

[TG09] TRICOCHE X., GARTH C.: Topological methods for visualizing vortical flows. In Mathematical Foundations of Scientific Visualization, Computer Graphics, and Massive Data Exploration, Farin G., Hege H.-C., Hoffman D., Johnson C. R., Polthier K., Rumpf M., (Eds.), Mathematics and Visualization. Springer Berlin Heidelberg, 2009, pp. 89-107. 10.1007/b106657_5. 2

[Val06] Vallis G. K.: Atmospheric and Oceanic Fluid Dynamics. Cambridge University Press, Cambridge, UK, 2006. 4

[VV92] Villasenor J., VinCENT A.: An algorithm for space recognition and time tracking of vorticity tubes in turbulence. CVGIP: Image Understanding 55, 1 (1992), 27 - 35. 2

[WAB06] Waugh D. W., Abraham E. R., Bowen M. M.: Spatial variations of stirring in the surface ocean: A case study of the tasman sea. J. Phys. Oceanogr.36, 3 (2006), 526 - 542. 2

[WF11] Williams R., Follows M.: Ocean Dynamics and the Carbon Cycle: Principles and Mechanisms, first ed. Cambridge University Press, 2011. 2

[WPB*11] Williams S., Petersen M., Bremer P.-T.,
Hecht M., Pascucci V., Ahrens J., HlawitschKa M., HAMANN B.: Adaptive extraction and quantification of geophysical vortices. Visualization and Computer Graphics, IEEE Transactions on 17, 12 (dec. 2011), 2088 -2095. 1, 2, 3

[WWH07] Williams R. G., Wilson C., Hughes C. W.: Ocean and Atmosphere Storm Tracks: The Role of Eddy Vorticity Forcing. Journal of Physical Oceanography 37 (2007), 2267. 8

[YNQ*10] Yim B. Y., NoH Y., QIU B., You S. H., Yoon J. H.: The vertical structure of eddy heat transport simulated by an eddy-resolving ogcm. Journal of Physical Oceanography 40, 2 (2010), 340-353. 2 